Dom. Cien., ISSN: 2477-8818

Vol. 4, núm.3., jul, 2018, pp. 313-333

\title{
La asignación presupuestaria y su incidencia en la Administración Pública
}

\author{
The budgetary allocation and its incidence in the Public Administration \\ A dotação orçamental e a sua incidência na Administração Pública
}

\author{
Walter M. Mendoza-Zamora ${ }^{\mathrm{I}}$ \\ uleam_eco_@outlook.com \\ Viviana M. Loor-Carvajal II \\ viviana.loor@hotmail.com \\ Ginger E. Salazar-Pin III \\ gingersalazar2604@hotmail.com \\ Dayan A. Nieto-Parrales IV \\ dayan.nieto@hotmail.com
}

Recibido: 27 de abril de $2018 *$ Corregido: 18 de junio de $2018 *$ Aceptado: 03 de julio de 2018

I. Magister en Gestión Empresarial, Ingeniero Comercial, Docente de la Universidad Estatal del Sur de Manabí, Jipijapa, Ecuador.

II. Magister en Administración Publica mención Desarrollo Institucional; Economista; Docente de la Universidad Estatal del Sur de Manabí, Jipijapa, Ecuador.

III. Magister en Contabilidad y Auditoría; Ingeniera Comercial; Docente de la Universidad Estatal del Sur de Manabí, Jipijapa, Ecuador.

IV. Maestrante Ingeniera Comercial, Docente Universidad Estatal del Sur de Manabí, Jipijapa, Ecuador. 


\section{Resumen}

La asignación presupuestaria es un instrumento que permite planificar, organizar, dirigir y controlar cada uno de recursos del Estado, el mismo debe cumplirse de manera obligatoria con el fin de gestionar y programar los ingresos y egresos para el adecuado financiamiento público. El análisis de los elementos que comprende el presupuesto general del Estado manifiesta la estimación de los recursos financieros que posee, estos deben ser directamente administrados por el Gobierno y sus instituciones a través del Ministerio de Economía y Finanzas. La planificación y ejecución de las asignaciones presupuestarias tiene como objetivo distribuir de forma equitativa las riquezas nacionales mediante planes estratégico u operativos que cumplan con las normativas vigentes y contribuyan al bienestar de la sociedad. La Administración Pública del Ecuador está encargada de canalizar adecuadamente las demandas sociales y satisfacer a través de la transformación de los recursos públicos las necesidades colectivas, del tal manera que ponga en contacto directo la ciudadanía con el gobierno, además es un sistema de gestión y calidad que sigua lineamientos para alcanzar un progreso donde se evidencie cambios laborales y sociales que beneficie al sector público. La gestión administrativa que realice cualquier funcionario o servidor público acorde a la asignación presupuestaria que presenten las instituciones públicas del Estado tienen que ser expresadas con claridad, de tal manera que pueda ser entendida por cada una de las organizaciones del Estado y de la sociedad, mediante informes públicos que muestren los resultados de su ejecución.

Palabras Claves: Financiamiento; Presupuesto; Recursos; Finanzas; Administración; Gestión; Instituciones; Ejecución. 


\begin{abstract}
The budget allocation is an instrument that allows to plan, organize, direct and control each of the State's resources, it must be complied with in order to manage and schedule the revenues and expenditures for adequate public financing. The analysis of the elements included in the general budget of the State shows the estimation of the financial resources it has, these must be directly administered by the Government and its institutions through the Ministry of Economy and Finance. The objective of planning and executing budget allocations is to distribute national wealth equitably through strategic or operational plans that comply with the regulations in force and contribute to the welfare of society. The Public Administration of Ecuador is in charge of adequately channeling social demands and satisfying, through the transformation of public resources, collective needs, in such a way that it puts citizens in direct contact with the government, it is also a management and quality system that follows guidelines to achieve progress that evidences labor and social changes that benefit the public sector. The administrative management carried out by any official or public servant according to the budgetary allocation presented by the public institutions of the state must be clearly expressed, in such a way that it can be understood by each of the State and society's organizations, through public reports that show the results of its execution.
\end{abstract}

Keywords: Financing; Budget; Means; Finance; Administration; Management; Institutions Execution. 


\section{Introducción.}

El Sistema Nacional de Finanzas Publicas de la República del Ecuador se basa en un conjunto de normas, procesos, actividades, políticas y operaciones. Las diversas entidades y organismos del sector público deben gestionar con el fin de programar los ingresos y egresos para un adecuado financiamiento de los recursos públicos, según el Plan Nacional de Desarrollo y las políticas públicas establecidas en la Ley.

El ciclo presupuestario del Ecuador debe cumplirse de manera obligatoria según el Código Orgánico de Planificación y Finanzas Publicas del Ecuador para las entidades que conforman el ámbito del Presupuesto General del Estado bajo la orientación de los servidores responsables, los mismos que deben seguir los procedimientos para la programación, formulación, aprobación, ejecución, evaluación, clausura y liquidación de los presupuestos públicos, según los programas y proyectos definidos en el marco del Plan Nacional de Desarrollo. (Ecuador, 2010)

La provincia de Manabí es una de las jurisdicciones que tiene mayor presupuesto para la ejecución de proyectos de desarrollo e inversión.

Peñaherrera, (1987) menciona: “la Gestión de la Administración Pública no ha sido producto de la participación equilibrada de las diferentes zonas de la patria, lo cual ha gravitado en un excesivo grado de centralización no necesariamente consecuente con la naturaleza unitaria de la organización política del Estado ecuatoriano.” (Peñaherrera, 1987)

Según (Sanipatin, 2017): Las instituciones públicas tienen la responsabilidad de cumplir con los procesos presupuestarios de acuerdo con cada una de las obligaciones ya que ahí radica la

\footnotetext{
316 Vol. 4, núm. 3, julio 2018, pp. 313-333
Walter M. Mendoza-Zamora; Viviana M. Loor-Carvajal; Ginger E. Salazar-Pin; Dayan A. NietoParrales
} 
calidad de gasto y la responsabilidad de los controles interno para la seguridad financiera y contable de la institución.

Los objetivos, políticas y metas establecidos por las entidades que conforman el ámbito del Presupuesto General del Estado contribuirán al progreso económico del país, de tal manera que los servidores encargados del manejo de la administración presupuestaria planificaran, organizaran, ejecutaran y controlaran cada uno de los procesos presupuestarios con el fin de mejorar la eficiencia asignativa y productiva del gasto público.

\section{Desarrollo}

\section{Materiales y métodos}

El trabajo desarrollado fue en el periodo de noviembre 2017 a marzo 2018 en la Universidad Estatal del Sur de Manabí, se realizaron investigaciones sobre la Asignación presupuestaria, así como también la incidencia que esta tiene en la Administración Pública.

Se realizó la investigación en base al método deductivo que permitió el alcance de los hechos más relevantes acorde a la programación y formulación del presupuesto, la programación de la ejecución presupuestaria, los responsables administrativos de cada unidad ejecutora y la evaluación de la gestión presupuestaria y el método bibliográfico, aplicado en el proceso de extracción de información en documentos web o sitios web, que permitieron localizar y seleccionar la información precisa de entre toda la masa documental que existe, logrando así el alcance de la investigación:

\footnotetext{
317. 4, núm. 3, julio 2018, pp. 313-333
Walter M. Mendoza-Zamora; Viviana M. Loor-Carvajal; Ginger E. Salazar-Pin; Dayan A. Nieto-
Parand Parrales
} 
La perspectiva del trabajo es cualitativa la misma para conocer de qué manera se lleva a cabo la gestión administrativa con relación a las asignaciones presupuestarias, y así determinar las verdades generales existentes

\section{Presupuesto}

(Ryckman, 2018) Menciona: "Un presupuesto es un plan financiero para estimar los ingresos y gastos de un período específico de tiempo".

\section{Presupuesto General del Estado Ecuatoriano}

El Presupuesto General del Estado es la estimación de los recursos financieros que tiene el Ecuador es el medio regulador y controlador de la economía y finanzas mediante el cual se debe manejar los gastos, inversiones y distribución participativa que debe realizar el Gobierno Central en función de los ingresos previstos para el año de su ejercicio de acuerdo con la Constitución y las leyes vigentes en el año de su uso. (Baquerizo, 2010)

El Presupuesto del Gobierno Central (PGC) es la parte del Presupuesto General del Estado (PGE) directamente administrada por el Gobierno y sus instituciones, a través del Ministerio de Finanzas. 


\section{Grafico 1 Asignación Presupuestaria}

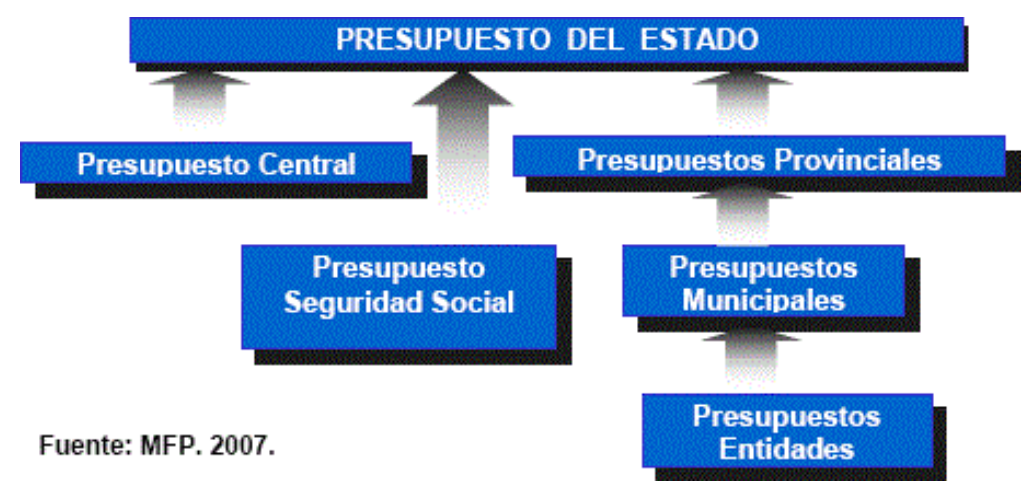

Clasificador presupuestario de ingresos y gastos

El clasificador presupuestario es una herramienta que posee un sistema integrado de información financiera, que permite ordenar la información según las disposiciones legales.

Ingresos del Presupuesto General del Estado

Los ingresos son los recursos que obtiene el Estado por la recaudación de tributos como el Impuesto a la Renta, el IVA; por la venta de bienes, como del petróleo y sus derivados; transferencias y donaciones que se reciben; el resultado operacional de las Empresas Públicas entre otros. Todos estos ingresos se destinarán para cubrir las obligaciones contraídas para la prestación de bienes y servicios públicos. (Ecuador, 2010)

Los ingresos del Presupuesto General del Estado son la estimación de los recursos financieros que tiene el Ecuador, es decir, los bienes y servicios que este brinde a la ciudadanía con el fin de satisfacer las necesidades y mantener un orden económico. 
Los ingresos se clasifican en:

- Ingresos permanentes.

- Ingresos no permanentes.

Los ingresos permanentes son aquellos ingresos que se mantienen en un periodo de tiempo ya que son recursos que recibe el Estado a través de sus instituciones de manera continua ya que los ingresos permanentes no ocasionan disminución de los activos públicos. (Ecuador, 2010)

\section{Grafico 2 Componentes del P.G.E}
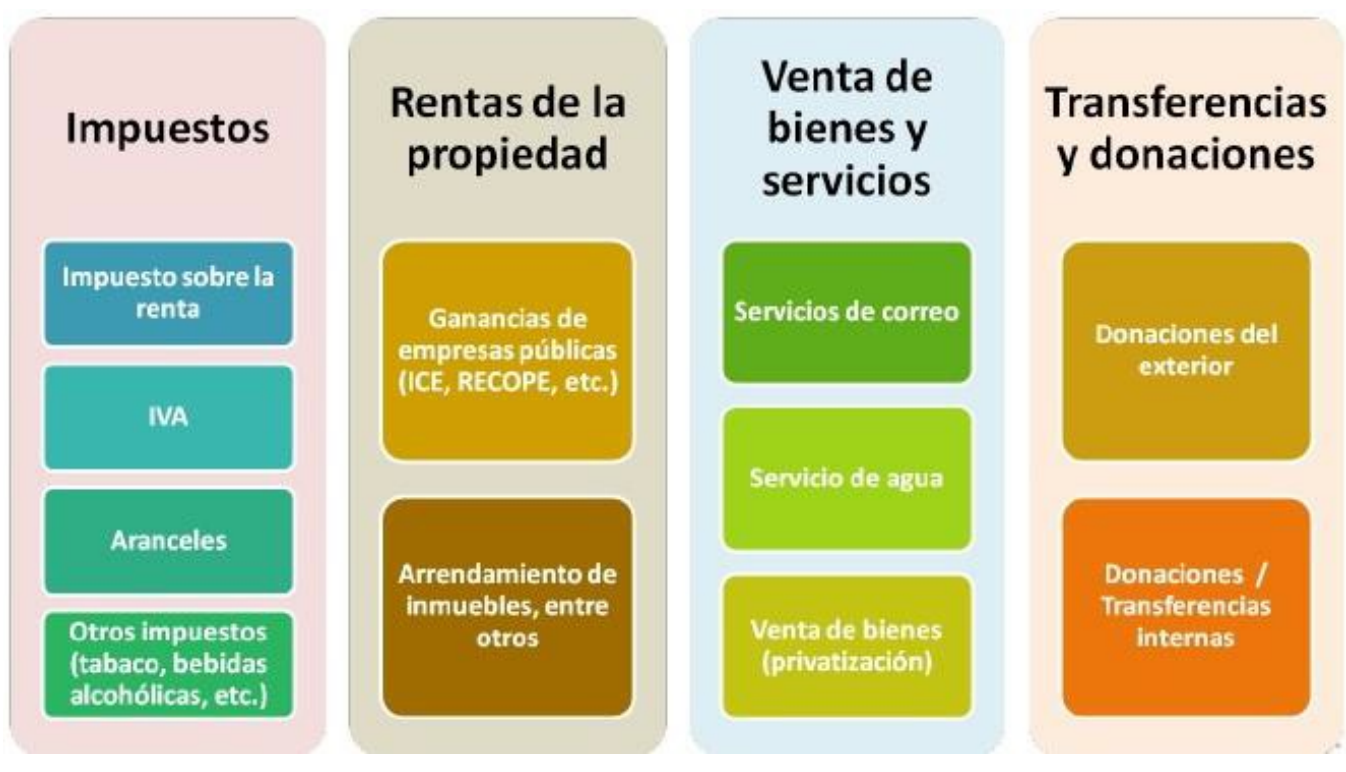

Fuente: Slideshare.net

Elaborado Por: Lenin Quillisimba

"Los ingresos no permanentes son aquellos recursos que el Estado recibe a través de sus instituciones estos ingresos son no predecibles en el tiempo por lo que se definen ingresos petroleros". (MEF, 2010) 
Egresos del presupuesto general del estado

Los gastos del presupuesto general del Estado son aquellos que ayudan al desarrollo de las actividades administrativas y de funcionamiento o que expresamente sirvan de financiamiento de inversión.

Estos se clasifican en

- $\quad$ Gastos permanentes. -

Son aquellos recursos que el Estado realiza para que las instituciones públicas puedan desarrollar sus actividades administrativas y de funcionamiento público, estos permiten la provisión continua de bienes y servicios a la sociedad.

- Gastos no permanentes. -

"Es todo aquel egreso que el Estado realiza de manera temporal por una situación específica, excepcional o extraordinaria y que no requieren continuidad en el tiempo. Pueden originar incremento de activo". (Alianza pais, 2015)

\section{El ciclo presupuestario}

Se conoce como un ciclo el inicio y final de diversas actividades, el Ministerio de Finanzas Publica del Estado ecuatoriano define al ciclo presupuestario como la programación y clausura de la ejecución presupuestaria, el mismo que tiene varias etapas que son:

- Programación

- $\quad$ Formulación

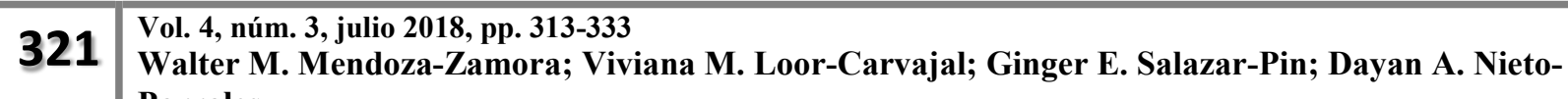
Parrales
} 
- Aprobación

- $\quad$ Ejecución

- $\quad$ Seguimiento y Control

- $\quad$ Clausura

- Liquidación

\section{Programación}

Es la primera fase del ciclo presupuestario, donde se especifican los objetivos y metas señalados en la planificación del Estado que se hace para cuatro años y por eso se lo denomina Presupuesto Plurianual.

"Tiene como propósito determinar los recursos reales y monetarios necesarios para lograr los objetivos y metas establecidos en los planes institucionales”. (Castillo \& Flor, 2010)

\section{Formulación}

Es la fase del proceso en la que se articulan las propuestas de acción y producción de los entes demandantes de recursos fiscales con los catálogos y clasificadores presupuestarios. Representa la estructura formal del presupuesto en los términos técnicos que el ente rector. ( Manual del Módulo de Programación , 2017)

Además, recoge los requerimientos de las instituciones comprendidas en el Presupuesto General del Estado, con la finalidad de apoyar al cumplimiento del Plan Nacional del Buen Vivir, así como lo establecido en el escenario macroeconómico.

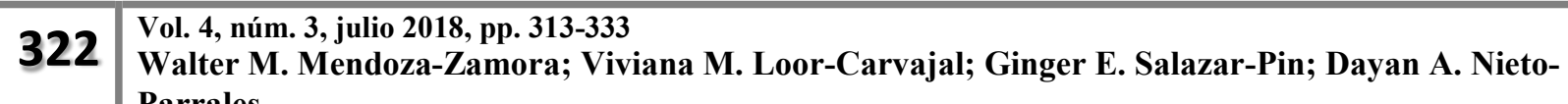
Parrales
} 
Aprobación

Las entidades del sector público no financiero cuyos presupuestos conforman el Presupuesto General del Estado, remitirán al Ministerio de Economía y Finanzas, hasta el 30 de junio de cada año, las proformas presupuestarias institucionales para su análisis y aprobación, con el fin de integrarlas y consolidarlas en la Proforma del Presupuesto General del Estado que será puesta a consideración del Presidente de la República, previo a su envío a la Asamblea, Luego, la Asamblea la reenvía a la Presidencia de la República para que en un tiempo de diez días acepte las observaciones, haga una nueva propuesta o se ratifique en la propuesta original.

La Asamblea Nacional, en los diez días siguientes, podrá ratificar sus observaciones en un solo debate, con el voto de dos tercios de sus integrantes; de lo contrario, entrará en vigencia la proforma presupuestaria enviada, a la cual se la denominará: Presupuesto Inicial Aprobado, y entrará en vigencia a partir del 1 de enero, sin prejuicio de su publicación en el Registro Oficial. (Enciclopoedia Juridica, 2014)..

\section{Ejecución}

Comprende el conjunto de acciones destinadas a la utilización de los recursos humanos, materiales y financieros asignados en el presupuesto con el propósito de obtener los bienes y servicios en la cantidad, calidad y oportunidad previstos en el mismo.

La ejecución presupuestaria se realizará sobre la base de las políticas establecidas por el ente rector de la administración financiera del Estado y comprende la formulación, seguimiento y evaluación de la programación de la ejecución física y financiera presupuestaria, la programación de caja y las modificaciones al presupuesto. 
Seguimiento y Control

El seguimiento y evaluación es una medición de resultados físicos y financieros obtenidos y los efectos que estos han producido; así como el análisis de variaciones financieras.

\section{¿Quién hace la evaluación?}

El Ministerio de Finanzas, de manera simultánea a la Ejecución Presupuestaria, es la entidad que realiza el seguimiento y evaluación financiera a las instituciones comprendidas en el Presupuesto General del Estado, las que a su vez internamente realizan el mismo proceso, pero con las empresas u organizaciones contratadas para brindar bienes y servicios.

\section{Clausura}

(Rumiñahui-Aseo.Emp, 2012) Menciona: Los presupuestos del sector público se clausurarán el 31 de diciembre de cada año. Después de esa fecha los ingresos que perciban se considerarán como del presupuesto vigente al momento que se cobren. Después del 31 de diciembre no se podrán contraer compromisos ni obligaciones que afecten al presupuesto del ejercicio fiscal anterior.

Las obligaciones de cobro y pago que quedaron pendientes al 31 de diciembre serán reconocidas en el nuevo período fiscal.

\section{Liquidación}

Es la fase del ciclo presupuestario que corresponde a la elaboración y exposición, al nivel consolidado, de la ejecución presupuestaria registrada a la clausura del ejercicio fiscal anua

\footnotetext{
324 Vol. 4, núm. 3, julio 2018, pp. 313-333
Walter M. Mendoza-Zamora; Viviana M. Loor-Carvajal; Ginger E. Salazar-Pin; Dayan A. NietoParrales
} 
La liquidación del Presupuesto del Gobierno Central se expedirá por acuerdo del Ministro de Finanzas, hasta el 31 de marzo del año siguiente y debe arrojar los siguientes resultados: - Déficit o Superávit Presupuestario

- Déficit o Superávit de Caja.

- Cuenta Ahorro Inversión Financiamiento - CAIF.

La liquidación refleja los resultados definitivos que dan cuenta del uso de los recursos por parte del Gobierno Central durante el año.

Desarrollo de asignaciones presupuestarias

La Constitución de la República del Ecuador (artículos 294 y 295) establece que la Función Ejecutiva, elabore cada año la proforma presupuestaria anual y la programación presupuestaria cuatrianual, que serán remitidas a la Asamblea Nacional para aprobación durante los primeros noventa días de su gestión y en los años siguientes sesenta días antes del inicio del año físcal respectivo. En sus artículos 280 y 293 señala que la programación y ejecución del Presupuesto General del Estado y la inversión y asignación de recursos públicos se sujetarán al Plan Nacional de Desarrollo.

Las instituciones del sector público realizaran una planificación donde reflejen sus necesidades básicas que les permita el desarrollo adecuado de la prestación de bienes y servicios a la población.

1.- El Gobierno Central tiene su propio presupuesto. Antes debemos entender que al decir Gobierno Central hablamos de la Presidencia de la República, Vicepresidencia de la República,

\footnotetext{
\begin{tabular}{l|l}
325 & $\begin{array}{l}\text { Vol. 4, núm. 3, julio 2018, pp. 313-333 } \\
\text { Walter M. Mendoza-Zamora; Viviana M. Loor-Carvajal; Ginger E. Salazar-Pin; Dayan A. Nieto- } \\
\text { Parrales }\end{array}$
\end{tabular}
} 
Ministerios y Secretarías de Estado y sus entidades adscritas, también están los presupuestos de las Funciones Legislativa (Asamblea Nacional), la Función Judicial (Corte Nacional de Justicia), además del Consejo Nacional Electoral y la Corte Constitucional.

2.- Organismos de control y regulación como la Contraloría, Superintendencia de Bancos y Compañías, Defensoría del Pueblo, etc. De la misma manera, instituciones autónomas y descentralizadas con personería jurídica, como Correos del Ecuador, Ferrocarriles, que son instituciones creadas por ley para prestar un servicio público, también tienen su presupuesto.

3.- Hay presupuesto de las instituciones de seguridad social creadas por ley, como el Instituto Ecuatoriano de Seguridad Social.

4.- Existe Presupuesto de los Ingresos Pre-asignados por ley (Código Orgánico de Organización Territorial - COOTAD), es decir, que anticipadamente según las necesidades de los municipios, prefecturas, juntas parroquiales se destina un porcentaje de los recursos del Presupuesto General del Estado para estos gobiernos locales.

5.- Existen presupuestos de los Fondos creados por ley, determinada mediante aprobación de la Asamblea Nacional para financiar programas y / o proyectos prioritarios.

El presupuesto ecuatoriano es distribuido según las prioridades que posea en cuanto a gastos e ingresos que posean las instituciones públicas tales son estos como: el gobierno central hasta aquellos que sirven para controlar según la ley, así mismo las instituciones que han sido creadas según la ley para la seguridad social.

Categoría de las asignaciones presupuestarias

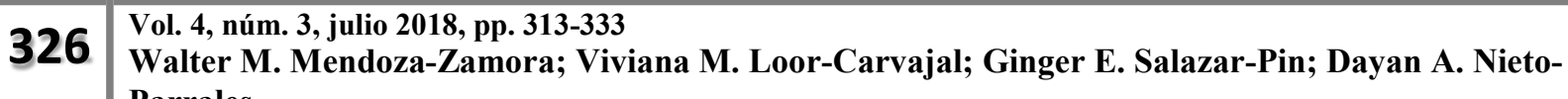
Parrales
} 
La división de un presupuesto se constituye en departamentos y unidades acorde a los programas o plan operativo de cada entidad pública.

Esto permite una fácil identificación de los recursos asignados a los programas y funciones específicas. Cada categoría se puede hacer de varias asignaciones presupuestarias, se hace referencia como elementos de línea, para las necesidades específicas necesarias para apoyar el funcionamiento general del departamento o programa.

Para poder realizar la asignación presupuestaria se debe contar con categorías que permitan identificar cada uno de los recursos esto no quiere decir que sean estáticas, al contrario, están sujetas a variaciones de acuerdo a la asignación presupuestaria que se realice según sean los programas establecidos.

\section{Administración pública ecuatoriana}

(Constitucion de la Republica del Ecuador, 2008) En la sección segunda art 227 indica: “La administración pública constituye un servicio a la colectividad que se rige por los principios de eficacia, eficiencia, calidad, jerarquía, desconcentración, descentralización, coordinación participación, planificación, transparencia y evaluación”.

La administración publica ecuatoriana se encarga de canalizar adecuadamente las demandas sociales y satisfacer a través de la transformación de los recursos públicos las necesidades colectivas, mediante la producción de bienes, servicios.

La administración pública ecuatoriana es regida bajo principios propios de la ley con el único fin de proporcionar bienestar a la ciudadanía, mantener la equidad entre las demandas sociales y

\footnotetext{
$327 \begin{aligned} & \text { Vol. 4, núm. 3, julio 2018, pp. 313-333 } \\ & \text { Walter M. Mendoza-Zamora; Viviana M. Loor-Carvajal; Ginger E. Salazar-Pin; Dayan A. Nieto- } \\ & \text { Parrales }\end{aligned}$ Parrales
} 
poder satisfacer cada una de ellas, las responsabilidades suministradas dentro de la administración pública constituyen un desarrollo sostenible y sustentable de la gestión de los recursos del Estado.

\section{Grafico 6: Canalización de la administración}

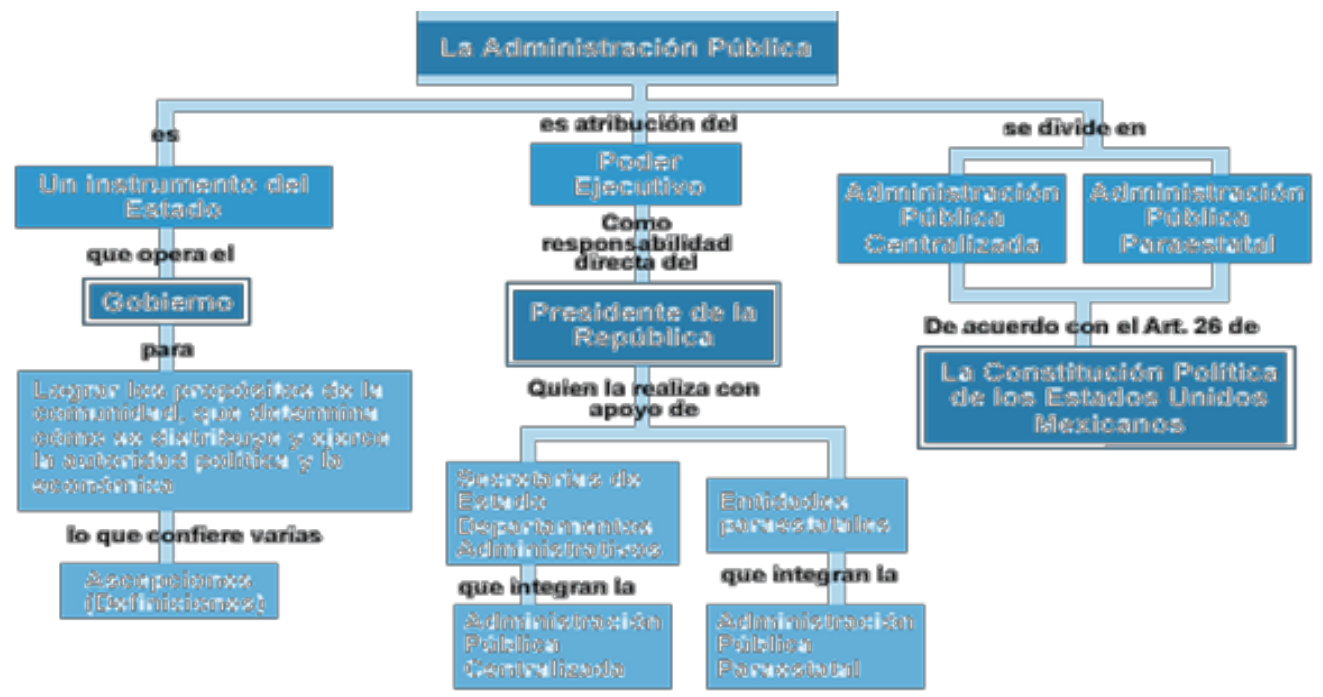

Fuente: Enciclopedia Financiera

Elaborado por: Enciclopedia Financiera

La administración pública y la asignación presupuestaria

El conjunto de recursos y obligaciones administrados por los entes financieros a través de los servidores públicos deberán regirse bajo el Plan Nacional de Desarrollo.

La unidad operativa desconcentrada cumple con las atribuciones y competencias para realizar las funciones administrativas y financieras. Los presupuestos se asignan acorde los planes

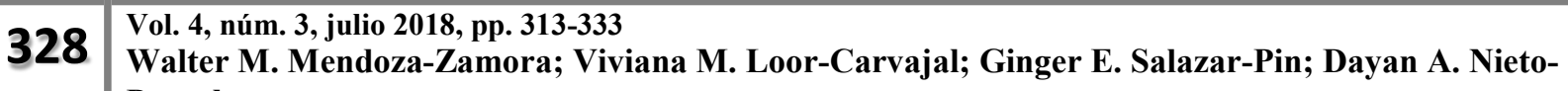
Parrales
} 
estratégicos y los planes operativos de cada entidad el mismo que deberá ser sustentado con los documentos de soporte de conformidad a las normas vigentes.

La gestión que proporcione la administración pública al momento de ejecutar una asignación presupuestaria deberá regirse bajo el Plan Nacional de Desarrollo en conformidad con las normas vigentes con el fin de mantener la equidad y evaluar las principales necesidades presentadas en planes estratégicos o planes operativos que manifiesten cada una de las instituciones públicas para la satisfacción de la ciudadanía

Las normas técnicas presupuestarias rigen para las instituciones del sector público no financiero y su aplicación es de carácter obligatorio. El presupuesto público asignado deberá regirse bajo principios de universalidad donde se contemple la totalidad de los gastos e ingresos que permita satisfacer al servicio de la comunidad, el mismo que debe ser programado según los requerimientos de recursos para conseguir los objetivos y metas que se programen en el horizonte anual y plurianual.

La gestión administrativa de la asignación presupuestaria se expondrá con claridad de forma que pueda ser entendida a todas las organizaciones del Estado y de la sociedad, mediante informe públicos que muestren los resultados de su ejecución.

La modificación presupuestaria será realizada por directores financieros cuando el prepuesto asignado afecte a un proyecto, programa o actividad el cual debe ser solicitado al Ministerio de Economía y Finanzas con los documentos que respalden la modificación.

\footnotetext{
329 Vol. 4, núm. 3, julio 2018, pp. 313-333
Walter M. Mendoza-Zamora; Viviana M. Loor-Carvajal; Ginger E. Salazar-Pin; Dayan A. NietoParrales
} 
Los responsables de la gestión financiera de las instituciones tienen la obligación de presentar un informe de evaluación financiera dela ejecución presupuestaria y un informe previo a la evaluación del avance de los programas en el periodo fiscal

El sector público está obligado legalmente a cumplir con la ejecución del presupuesto. Las autorizaciones presupuestarias tienen un carácter limitativo y fundamentado bajo normas y leyes que aseguren el logro de los objetivos previstos en el plan.

\section{Control del sistema nacional de presupuesto público}

El Ministerio de Finanzas en forma conjunta con la Contraloría General del Estado, emitirá las normas técnicas para implementar un sistema de control y evaluación del sistema nacional de presupuesto público. Dicho sistema, entre otros aspectos, comprenderá:

a) El examen y análisis permanente de las operaciones y registros de los activos, pasivos, ingresos, costos y gastos en general.

b) La medición de las realizaciones físicas y de los recursos financieros asignados en los programas autorizados.

c) El análisis de las variaciones entre lo programado y lo ejecutado y la determinación de sus causas.

d) La medición de los niveles de eficiencia, eficacia y económica en la gestión pública.

e) La legalidad que conllevan la ejecución de las actividades y proyectos, conforme a los ordenamientos legales vigentes.

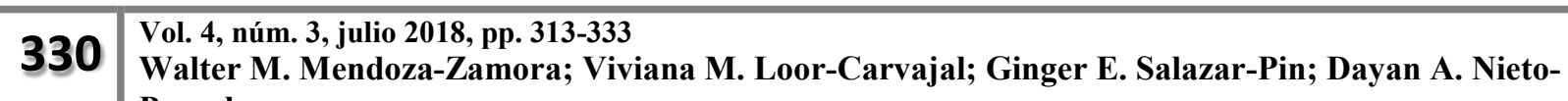
Parrales
} 
f) El análisis de la situación económica financiera.

g) La adopción de las medidas correctivas.

El sistema de control y evaluación que para estos efectos se determine, deberá ser compatible con el sistema de control de los recursos públicos establecido por la Contraloría General del Estado.

Los entes rectores de finanzas publicas en conjunto con la contraloría general del Estado, son las únicas instituciones que proveerán las leyes y normas para la correcta ejecución del presupuesto, los mismo que tendrán la disposición de realizar el seguimiento y evaluación de todos los movimientos contables de las instituciones públicas financieras o no financieras.

\section{Conclusiones.}

La asignación presupuestaria está comprendida por un plan financiero estratégico elaborado con la finalidad de indicar los niveles de recursos y así que a todas las instituciones se les presente un límite en cuanto a sus gastos, se encuentran sujetas a constantes monitoreo que aseguren que estos no incurran en un déficit debido a la mala administración de los mismos. La asignación presupuestaria cuenta con un periodo de desarrollo de doce meses para ello tiene categorías que especifican las necesidades así estas permiten el correcto funcionamiento de los programas.

Los presupuestos que los gobiernos elaboran para un determinado periodo fiscal son utilizados con el propósito de controlar y manejar efectivamente las partidas de gastos y de ingresos, por esto se hace imprescindible la clasificación de las partidas que avalan las cuentas de dicho presupuesto esto genera a su vez un mayor control y una mayor eficiencia a la hora de ponerlo en

\footnotetext{
331 Vol. 4, núm. 3, julio 2018, pp. 313-333
Walter M. Mendoza-Zamora; Viviana M. Loor-Carvajal; Ginger E. Salazar-Pin; Dayan A. NietoParrales
} 
práctica, ya que una clasificación adecuada genera un equilibrio perfecto y a su vez una herramienta eficaz para la administración.

El Presupuesto del Estado Ecuatoriano es una herramienta útil y dinámica la cual cumple con varias etapas, denominadas ciclo presupuestario, el cual refleja varias acciones desde su inicio hasta su final, para ello cuenta con etapas con las que cumple este ciclo y son: programación, formulación, aprobación, ejecución, evaluación y seguimiento, clausura y liquidación, las mismas que comprenden las metas y objetivos que estarán definidos en los proyectos y programas recogiendo los requerimientos de cada institución publicad con la finalidad de proponer límites para sus gastos y le puedan dar el cumplimiento necesario a la planificación del Estado.

Como Administración Pública se identifica al segmento del Estado que tiene a su cargo la realización de la función administrativa, equivalente en suma a realizar, ejecutar, desarrollar o instrumentar el programa político y a los organismos de la Administración Central del Estado corresponde el ejercicio de la actividad ejecutiva y administrativa en el marco de sus respectivas competencias, convirtiéndose de hecho en el medio por excelencia a través del cual el Estado ha de procurar la mayor parte de los objetivos que se plantean también ponen en contacto directo a la ciudadanía con el poder político, satisfaciendo los intereses públicos de forma inmediata, por contraste con los poderes legislativo y judicial, que lo hacen de forma mediata.

\section{Referencias.}

Manual del Módulo de Programación . (2017). Obtenido de https://www.mef.gob.pe/contenidos/siaf/manuales/MU_MPF_2017_GN_GR.pdf

Alianza pais. (23 de 11 de 2015). Obtenido de https://www.alianzapais.com.ec/2015/11/23/ec-449tema-ingresos-egresos-permanentes-no-permantes/

Baena, E. (11 de 11 de 2009). Aprendeconomia. Obtenido de https://aprendeconomia.com/2009/11/11/3-el-sector-publico/ 
Baquerizo, C. (02 de 02 de 2010). Desde mi Trinchera. Obtenido de http://www.desdemitrinchera.com/2010/02/02/presupuesto-general-del-estado/

Bonilla, María; Molina, José; Morales, Frank. (14 de 04 de 2006). Obtenido de http://frankmorales.webcindario.com/trabajos/planificacion.html

Castillo, G., \& Flor, M. (2010). Sistema de presupuesto con aplicación a la Casa de la Cultura Ecuatoriana Benjamin Carrion núcleo Morona Santiago. MACAS.

Constitucion de la Republica del Ecuador. (2008). Quito.

Economia, E. d. (2018). Obtenido de http://www.enciclopediafinanciera.com/

Enciclopoedia Juridica. (2014). Obtenido de http://www.enciclopediajuridica.biz14.com/d/aprobaci\%C3\%B3n/aprobaci\%C3\%B3n.htm

Finanzas, M. d. (31 de 01 de 2018). Ministerio de Economia y Finanza. Obtenido de http://www.finanzas.gob.ec/el-presupuesto-general-del-estado/

Georges, V. (01 de 01 de 2007). Blogspot.com. Obtenido de http://irmasusana.Blogspot.com/2007/01/administracion-pblica.html

Hugo, T. (1995). La Administracion Publica del Ecuador. Espae.

Ivette Flores Jiménez \& Ruth Flores Jimenez. (s.f.). Obtenido de https://www.uaeh.edu.mx/investigacion/estl/LI_AdmninEst/Ruth_Flores/articulo_planificaci on.pdf

J.C, Q. P. (05 de 2009). eumed.net. Obtenido de http://www.eumed.net/ce/2009b/jcqp2.htm

Jorge, B. R. (2005). Presupuesto, enfoque de planeacion y control de recursos. Colombia: McGraw Hill.

Julian Perez Porto \& Maria Merino. (2014). Obtenido de https://definicion.de/deuda-publica/

Ley Organica del Servicio Publico. (2005).

Nicolas, M. (2015). El Principe, Obras Politicas. La Habana: Ciencias Sociales.

Ordóñez, H. J. (24 de 11 de 2005). DerechoEcuador. Obtenido de https://www.derechoecuador.com/la-administracion-publica

Peñaherrera, D. C. (1987). Administracion Publica Ecuatoriana. Quito-Ecuador: Santiago Escobar.

Perez, A. (21 de 07 de 2014). Gestiopolis. Obtenido de https://www.gestiopolis.com/laadministracion-publica-su-relacion-con-el-estado/

Públicas., C. O. (2008). Obtenido de https://es.slideshare.net/PresidenciaEc/ec449-tema-ingresosegresos-permanentes-no-permantes

Rumiñahui-Aseo.Emp. (31 de 12 de 2012). Obtenido de http://www.ruminahui-aseo.gob.ec/wpcontent/uploads/2013/08/Liquidaci\%C3\%B3n-Presupuestaria-2012.pdf

Ryckman, M. L. (03 de 01 de 2018). La voz de houston. Obtenido de https://pyme.lavoztx.com/ques-una-asignacin-presupuestaria-12718.html

Sanipatin, D. (03 de 2017). Repositorio Digital Unesum. Obtenido de http://repositorio.unesum.edu.ec/handle/53000/701 